\title{
Can disorder act as a chemical pressure? An optical study of the Hubbard model
}

\author{
Himadri Barman, ${ }^{1, *}$ Mukul S. Laad,${ }^{1, \dagger}$ and Syed R. Hassan ${ }^{1, \ddagger}$ \\ ${ }^{1}$ Institute of Mathematical Sciences, Taramani, Chennai 600113, India
}

(Dated: March 13, 2021)

\begin{abstract}
The optical properties have been studied using the dynamical mean-field theory (DMFT) on a disordered Hubbard model. Despite the fact that disorder turns a metal to an insulator in high dimensional correlated materials, we notice that it can enhance certain metallic behavior as if a chemical pressure is applied to the system resulting in an increase of the effective lattice bandwidth (BW). We study optical properties in such a scenario and compare results with experiments where the BW is changed through chemical doping and obtain remarkable similarities vindicating our claim. We also make a point that these similarities differ from some other forms of BW tuned optical effects.

PACS numbers: 71.27.+a, 71.10.Fd, 71.10.-w 71.10.Hf, 71.10.Fd 71.30.+h, 72.80.Ng 78.30.Ly 78.20.-e
\end{abstract}

Optical studies have driven a huge attention towards understanding interaction effects on strongly correlated electronic materials (SCEMs), specifically after the discovery of high temperature superconductors [1, 2]. For the frequency $(\omega)$ dependent complex optical conductivity $\sigma(\omega)=\sigma_{1}(\omega)+i \sigma_{2}(\omega)$, real $\left(\sigma_{1}\right)$ and imaginary $\left(\sigma_{2}\right)$ parts of it provide much information to probe properties beyond the Drudé paradigm of optical conductivity for SCEMs. Recently many dynamical quantities related to optical conductivity, particularly the effective carrier density, scattering rate and dynamical effective mass have been found to be useful in understanding correlated metallic phase in cuprates [1], pnictides [3, 4], $\mathrm{V}_{2} \mathrm{O}_{3}$ [5], $\mathrm{VO}_{2}$ [6], organic conductors [7], ruthenates [8$10]$, and other correlated materials [11].

It is known that pressure or doping the transition metals with ions of equal valency but a different size (chemical pressure) leads to a change in the effective bandwidth (BW) of a transitional metal oxide (TMO). In the halffilling carrier concentration such a change may give rise to a Mott metal-to-insulator transition (MIT). Such a transition is often dubbed bandwidth-controlled MIT (BCMIT) [12]. Though extensive optical studies have been performed on myriads of TMOs and other SCEMs [11], studies of disorder-effect on them occupy limited volumes in the literature, both in experiments and theories. Recently Radonjić et al [13] studied a disordered Hubbard model following the X-ray irradiation induced disorder in $\kappa$-BEDT organic conductor [14], which is a typical twodimensional Mott insulator. However, the authors limited their investigation to the extended metalicity due to BW increase in presence of disorder, from their transport and optical results, while how the BW change affects several dynamic properties compared to the similar effect in clean systems remains unanswered. This sets up a motivation to investigate the mentioned optical properties and relate them to BW-controlled physics.

The site-disordered Hubbard model is written as

$\hat{H}=-\sum_{\langle i j\rangle . \sigma} t_{i j} c_{i \sigma}^{\dagger} c_{j \sigma}+U \sum_{i} n_{i \uparrow} n_{i \downarrow}+\sum_{i \sigma}\left(\epsilon_{c}-\mu+v_{i}\right) n_{i \sigma}$ where $c_{i \sigma}^{\dagger} / c_{i \sigma}$ is the electron creation/annihilation operator with spin $\sigma$ at site $i, t_{i j}$ indicates the amplitude of hopping from site $i$ to $j$ (typically $t_{i j}=t \forall i, j$ ), $U$ is the onsite Coulomb interaction, $\mu$ and $\epsilon_{c}$ are chemical potential and orbital energy of the electrons in clean system, and $v_{i}$ is the disorder potential at site $i$. The model has been addressed by several authors, particularly within the framework of dynamical mean-field theory (DMFT) [13, 15-19] where the spectral density shows a disorder driven MIT in a certain parameter regime. However, the optical properties comparatively received lesser attention and we investigate the dynamic properties studied in the clean system [7] in our disordered model.

Method : We solve Eq. (1) using the DMFT in which a correlated lattice model is mapped onto a single impurity Anderson model where the impurity is selfconsistently connected to a non-interacting fermionic bath [20]. Despite the lattice problem gets simplified in this way, the impurity model still requires many-body numerics to be solved and among many such existing methods [20] we employ the standard iterated perturbation theory (IPT) which is a second order perturbation around the Hartree-Fock (HF) self-energy : [21]

$$
\Sigma_{\mathrm{IPT}}(\omega)=\Sigma_{\mathrm{HF}}+\Sigma^{(2)}(\omega)
$$

with

$$
\begin{aligned}
\Sigma_{\mathrm{HF}} & =U\left\langle\hat{n}_{\sigma}\right\rangle=U n / 2 \\
\Sigma^{(2)}(\omega) & =\lim _{i \omega_{n} \rightarrow \omega} \frac{U^{2}}{\beta^{2}} \sum_{m, p} \mathcal{G}\left(i \omega_{n}+i \nu_{m}\right) \mathcal{G}\left(i \omega_{p}+i \nu_{m}\right) \mathcal{G}\left(i \omega_{p}\right)
\end{aligned}
$$

where $n$ is average occupancy, $\beta$ is inverse temperature, $i \omega_{n}, i \omega_{p}, i \nu_{m}$ are relevant Matsubara frequencies, and $\mathcal{G}$ is the Weiss Green's function of the non-interacting bath [20]. In practice, either of $\mathcal{G}$ or $\Sigma$ is guessed in the first iteration of the self-consistency loop. The selfconsistency condition in DMFT equates both the impurity and lattice self-energies and hence the same selfenergy in Eq. (2) is used to calculate the lattice Green's 
function $G(\omega)=\int d \epsilon D_{0}(\epsilon) /(\omega+\mu-\epsilon-\Sigma(\omega))$ where $D_{0}$ is the non-interacting lattice density of states and we choose the Bethe lattice in our work where $D_{0}(\epsilon)=$ $2 /(\pi t) \sqrt{\left.1-(\epsilon / t)^{2}\right]}$ implying non-interacting BW in our theory, $\mathcal{W}_{\text {th }}=2 t$. We also work in the half-filling case $(n=1, \mu=U / 2)$ and select a binary alloy distribution of the disorder potential $P\left(v_{i}\right)=(1-w) \delta\left(v_{i}\right)+w \delta\left(v_{i}-v\right)$ where $w$ is the weight for disordered sites and for the half-filling case $w=1 / 2$. The effect of disorder is treated through the coherent potential approximation (CPA) [13, 15, 22, 23], which gives rise to the following self-energy and Green's function.

$$
\begin{aligned}
& \Sigma_{\mathrm{CPA}}(\omega)=w v+\frac{w(1-w) v^{2}}{\omega-v(1-w)-\Delta(\omega)}, \\
& \mathcal{S}_{\mathrm{CPA}}(\omega)=\frac{1-w}{\omega-\Delta(\omega)}+\frac{w}{\omega-v-\Delta(\omega)}
\end{aligned}
$$

with $\Delta(\omega)$ defined through the Dyson Eq. $\Delta(\omega)=\omega+$ $\mu-v / 2-\Sigma(\omega)-G^{-1}(\omega), \Sigma(\omega)=\Sigma_{\mathrm{IPT}}+\Sigma_{\mathrm{CPA}}$. $\mathcal{G}_{\mathrm{CPA}}$ becomes the updated Weiss function $\left(\mathcal{G}=\mathcal{G}_{\mathrm{CPA}}\right)$ and hence feeds back to Eq. (4) in the DMFT self-consistency loop.

To determine the optical conductivity we use the standard expression based on the Kubo formalism : [21, 24]

$\sigma(\omega)=\sigma_{0} \int_{-\infty}^{\infty} d \omega^{\prime} F\left(\omega, \omega^{\prime}\right) \int_{-\infty}^{\infty} d \epsilon \Phi_{x x}(\epsilon) D_{\epsilon}\left(\omega^{\prime}\right) D_{\epsilon}\left(\omega^{\prime}+\omega\right)$.

where $\sigma_{0} \equiv 4 \pi e^{2} / \hbar$ ( $e$ and $\hbar$ being electronic charge and the reduced Planck's constant), $F\left(\omega, \omega^{\prime}\right) \equiv\left[f\left(\omega^{\prime}\right)-f(\omega+\right.$ $\left.\left.\omega^{\prime}\right)\right] / \omega$ and $\Phi_{x x}$ is called the transport function defined as

$$
\Phi_{x x}(\epsilon) \equiv \frac{1}{N} \sum_{\mathbf{k}}\left(\frac{d \epsilon_{\mathbf{k}}}{d k_{x}}\right)^{2} \delta\left(\epsilon-\epsilon_{\mathbf{k}}\right)
$$

where $\epsilon_{\mathbf{k}}$ is the momentum (k) dependent lattice dispersion. We choose $\Phi_{x x}^{\mathrm{HCL}}(\epsilon)=D_{0}(\epsilon)$ which is exact for the hypercubic lattice at infinite dimension and a reasonable approximation for the Bethe lattice that we consider in our calculations [25]. For simplicity, we express $\sigma(\omega)$ in the unit of $\sigma_{0}$, i.e. we set $\sigma_{0}=1$.

Results : We first look at the real part of the complex optical conductivity : $\sigma_{1}(\omega) \equiv \operatorname{Re} \sigma(\omega)$. Since disorder induces localization of electrons, and CPA effectively captures such an effect for disordered binary alloys [15], our zero temperature results show that the Drudé peak $\left(\sigma_{1}(0)\right)$ diminishes as disorder strength increases and finally disappears by opening an optical gap at $v>v_{c}$ $\left(v_{c} \simeq 0.5 U\right.$ at $U=2 t$, see Fig. 1(a).), signaling an MIT (see inset in Fig. 1(a) for the spectral densities reflecting the same). The Drudé peak becomes finite at finite temperature $(T)$ and slowly merges with the first absorption peak as temperature is raised. The optical gap formed at large disorder strength $(v=0.8 U)$ gets closed at high temperature $(T=0.1 t$, see Fig. $1(b))$. :

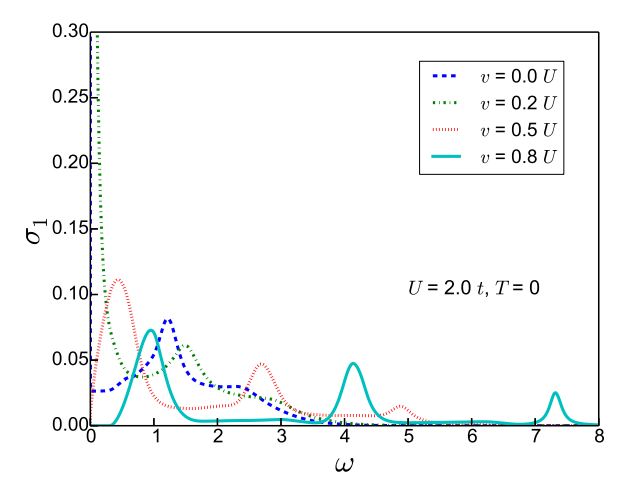

(a)

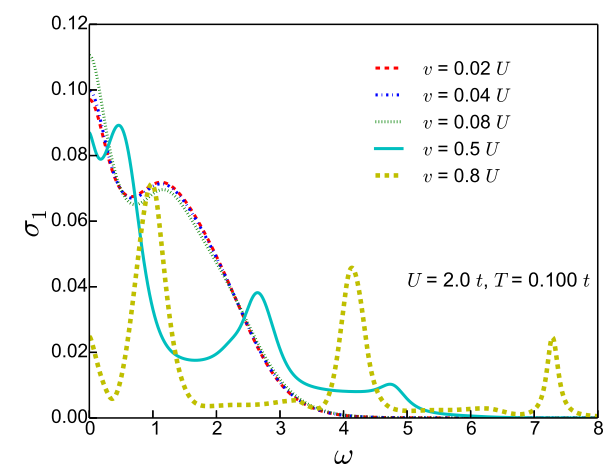

(b)

Fig. 1. Imaginary parts of optical conductivity as functions of frequency $(U=2 t)$ at (a) zero temperature and (b) finite temperature. The inset in (a) shows the corresponding spectral densities $A(\omega)=-\operatorname{Im} G(\omega) / \pi$.

Though this signifies transition from a good metal to bad metal, our interest sticks to the case where disorder leads to the change in the BW before it ends up with an Anderson-like insulating phase at $v>v_{c}$. This bears a close resemblance to the BC-MIT, where higher disorder strength effectively increases the lattice BW and following that it increases the quasiparticle weight $Z=\left.(1-\partial \Sigma / \partial \omega)\right|_{\omega=0}$ in the coherent regime [13, 15, 23].

Recently several optical measurements have been performed on the Br-doped organic conductor $\kappa$-BEDT [7] where particularly three kinds of optical property have been investigated, namely (i) effective carrier density $\left(N_{\text {eff }}(\omega)\right)$, (ii) dynamic scattering rate $(\tau(\omega))$, and (iii) effective optical mass $\left(m^{*}(\omega)\right)$. Following the $f$-sumrule $\int_{0}^{\infty} d \omega \sigma_{1}(\omega)=\pi n e^{2} /(2 m)$ for the Drudé optical conductivity of free electron metals, one can define the effective spectral weight or charge density $N_{\text {eff }}$, by performing cumulative sum (instead of full sum upto $\infty$ ) on $\sigma_{1}(\omega)$ [11]

$$
N_{\mathrm{eff}}(\omega) \equiv \frac{2 m_{\mathrm{opt}}}{\pi e^{2}} \int_{0}^{\omega} d \omega^{\prime} \sigma_{1}\left(\omega^{\prime}\right)
$$

where $m_{\text {opt }}$ is called the optical mass of electrons (equivalent to effective mass derived from the bandstructure 
but in presence of correlation now) for the generic Drudé theory [11].

The other two quantities are defined from the complex optical conductivity for the generalized Drudé model : $4 \pi \sigma(\omega, T)=\omega_{p}^{2} /\left[\tau^{-1}(\omega, T)-i m^{*}(\omega, T) / m_{\mathrm{opt}}\right]\left(\omega_{p}\right.$ is the plasma frequency), following which we find

$$
\begin{array}{r}
\frac{1}{\tau(\omega)}=\frac{\omega_{p}^{2}}{4 \pi} \operatorname{Re}\left[\frac{1}{\sigma(\omega)}\right], \\
\frac{m^{*}(\omega)}{m}=-\frac{\omega_{p}^{2}}{4 \pi \omega} \operatorname{Im}\left[\frac{1}{\sigma(\omega)}\right] .
\end{array}
$$

Effective carrier density - The main panel in Fig. 2(a) shows how $N_{\text {eff }}(\omega)$ changes with $\omega$ at various disorder strengths. As a generic trend, $N_{\text {eff }}(\omega)$ increases with $\omega$ as more charge carriers can be excited at higher optical energy. Increasing $v$ leads to increasing $\mathrm{BW}$ and hence

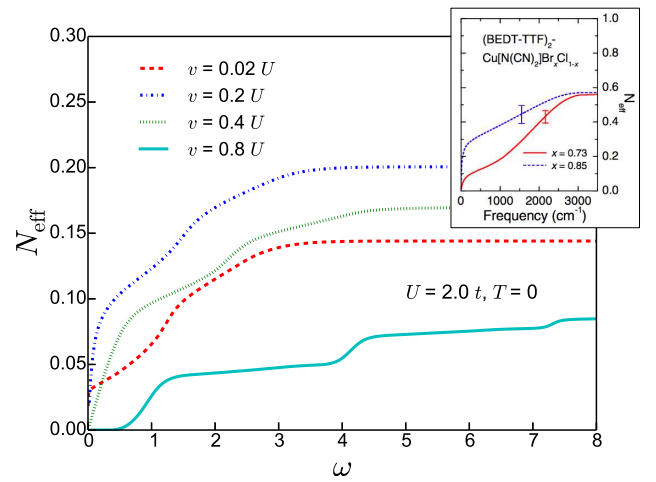

(a)

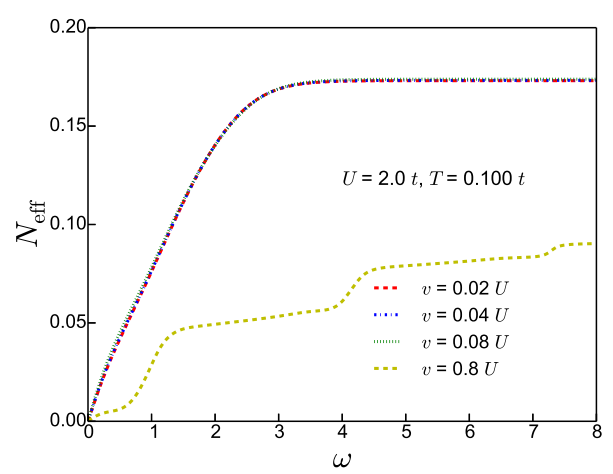

(b)

Fig. 2. (a) Effective carrier density $N_{\text {eff }}$ as functions of frequency $\omega$ at various disorder strengths at $U=2 t$. Inset shows experimental results at dopings $x=0.73$ and $x=0.85$ reproduced from 7 . Increase in $x$ leads to reduction of $U / t$ i.e. increase in effective BW. (b) $N_{\text {eff }}$ as functions of frequency $\omega$ at $T=0.1 t$.

the Drudé peak gets broadened (see Fig. 1(a)) resulting in more weight in the $f$-sumrule at low frequency. Since for small $v / U$, significant change solely happens around the Drudé peak $(\omega / t \lesssim 1)$ and hence below a critical value $v_{c}, N_{\text {eff }}(\omega)$ is higher at higher $v / U$. From Fig. 1(a) one can also speculate that slope change in the optical sum-rule should occur at the frequency where there is a significant feature (such as an absorption peak or shoulder) in $\sigma_{1}(\omega)$. Thus the peaks and shoulder at $\omega \simeq 1.4 t$ and 2.5t manifest change of slopes at the same values for $v=0.02 U$, while $U=2 t$. At high frequency, since there remains no charge carrier density to be excited: $\sigma_{1}(\omega \rightarrow \infty) \rightarrow 0, N_{\text {eff }}(\omega)$ 's value does not alter much and finally saturates. Similar trends have been noticed in experiments described in Ref. 7 (see inset of Fig. 2(a); $x$ implies doping concentration in the legend).

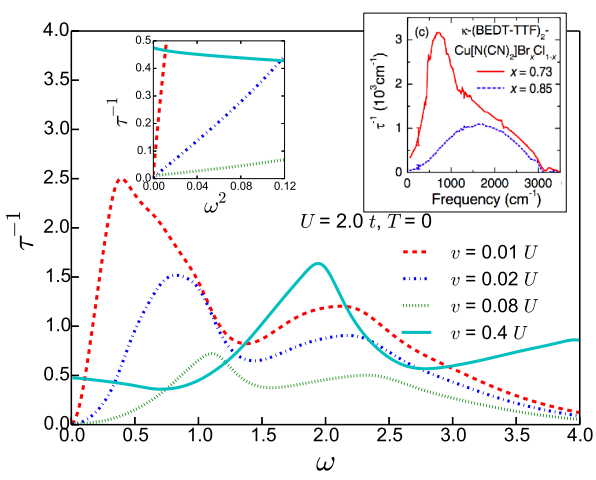

(a)

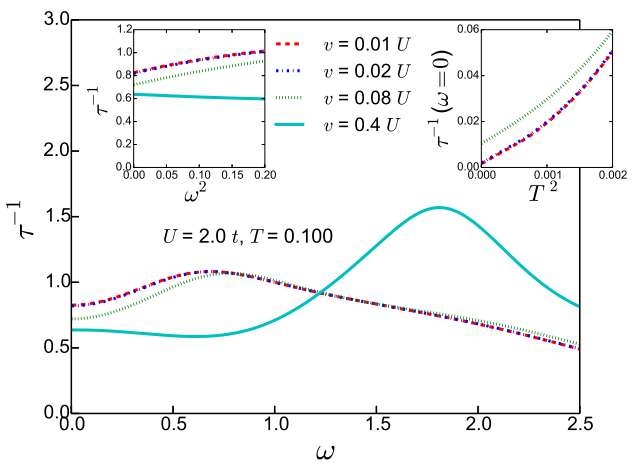

(b)

Fig. 3. Dynamic scattering rates as functions of frequency $(U=2 t)$ at (a) $T=0$ and at (b) $T=0.1 t$. The right inset in (a) shows similarities with the experiment reproduced from Ref. 7. The other insets show Fermi liquid frequency and temperature dependences and their breakdowns at $v=0.4 U$ and $T>0.01 t$.

The feature is more or less the same at finite temperature. However, at quite high temperature $(T>0.05 t)$ the Drudé peak significantly melts down and merges with absorption peaks resulting in featureless monotonically increasing $N_{\text {eff }}$ as a function of $\omega$ until it reaches the saturation at large $\omega$ (see Fig. 2(b)). In the insulating regime $\left(v>v_{c}\right), N_{\text {eff }}(\omega)=0$ for $\omega<\Delta_{\text {opt }}$ where $\Delta_{\text {opt }}$ is the optical gap. At higher $\omega, N_{\text {eff }}(\omega)$ starts increasing and forms plateaus at positions where the absorption peaks appear in $\sigma_{1}(\omega)$.

Dynamic scattering rate - Next we look at the dynamic 
scattering rate $\tau(\omega)$ as defined in Eq. (10). For a Fermi liquid, it measures the quasiparticle lifetime and it bears the following frequency-temperature $(\omega, T)$ dependence.

$$
\tau^{-1}(\omega, T)=A \omega^{2}+B(\pi T)^{2}
$$

where $A$ and $B$ are constants $[26,27]$. At low or zero temperature, for $v<v_{c}, \tau^{-1}$ depends on $\omega$ and $T$ in accord with Eq. (12) and hence signifies a Fermi-liquid metallic phase. The insets in Fig. 3 show the plots against $\omega^{2}$ and $T^{2}$ supporting the fact. For $v \geq v_{c} \simeq 0.4 U, \omega^{2}$ dependence and $T>0.01 t, T^{2}$ dependence get violated, insinuating breakdown of FL regime. At zero temperature a mid-infrared peak cum shoulder feature arises in the scattering rate while the shoulder is not much evident in the experiment reported in Ref. 7. However, the DMFT results in the same reference (BW tuned by $U / t$ ratio) contain the shoulder feature reinforcing the fact BW tuning by both $U / t$ and $v / U$ are of the same ilk. The peak position appears around $750 \mathrm{~cm}^{-1}$ and 1500 $\mathrm{cm}^{-1}$ respectively for Br-doping $x=0.73$ and $x=0.85$ respectively, which are $0.30 \mathcal{W}_{\mathrm{ex}}$ and $0.62 \mathcal{W}_{\mathrm{ex}}$ considering the experimental noninteracting $\mathrm{BW}, \mathcal{W}_{\mathrm{ex}}=0.3 \mathrm{eV}$ $\simeq 2419.66 \mathrm{~cm}^{-1}$ [7]. Similarly, though disorder is a different drive compared to the experiment, the peaks for $v=0.01 U$ and $v=0.08 U$ at $U=2 t$ occurs around $0.5 t=0.25 \mathcal{W}_{\text {th }}$ and $1.2 t=0.6 \mathcal{W}_{\text {th }}$ respectively, which are remarkably within the same energy range. Blueshifting of the peak due to increase in BW also agrees with the experiment. At sufficiently high temperature $(T=0.1 t)$ the shoulder feature disappears followed by a long universal tail extending to high frequency. After $v \geq 0.4 U$, the quasiparticle description entirely breaks down and $\tau^{-1}$ starts decreasing as frequency increases (see Fig. 3).

Effective optical mass - Optical mass is another interesting property that tells about the renormalization of the electronic mass as a combined effect of electronic bandstructure and correlation. Like in Ref. 7 the dynamical mass ratio $m^{*}(\omega) / m$ at low $\omega$ also decreases due to increase of the BW by rise in disorder strength. While the real part contribution $\tau^{-1}(\omega)$ of the conductivity in Eq. (10) gives rise to a peak feature, the contribution from its imaginary part (Eq. (11)) forms a dip in the midinfrared frequency range. In the experiment described in Ref. 7 the dip occurs around $1000 \mathrm{~cm}^{-1}\left(=0.413 \mathcal{W}_{\mathrm{ex}}\right)$ $1900 \mathrm{~cm}^{-1}\left(=0.785 \mathcal{W}_{\text {ex }}\right)$ for dopings $(x) 0.73$ and 0.85 respectively. Similarly for $v=0.01 U$ and $v=0.08 U$, the dips appear at $0.8 t=0.4 \mathcal{W}_{\text {th }}$ and $1.4 t=0.7 \mathcal{W}_{\text {th }}$ respectively, which are again in the same energy range. As the BW increases further due to increase in $v / U$, the dip experiences a blueshift and becomes shallower. At $v \geq 0.4 U$ the dip continues blueshifting, however, the ratio increases with frequency at low $\omega$ regime instead of decreasing, again signaling a breakdown of Fermi liquid coherence. At $T \geq 0.02 t, m^{*}(\omega) / m$ value drops down near $\omega=0$ and almost collapses for all frequency range for various $v$ 's less than $0.4 U$ (see Fig. 4(b)).

Other BW controlling factors - There exist several factors which can control the effective BW of a cor-

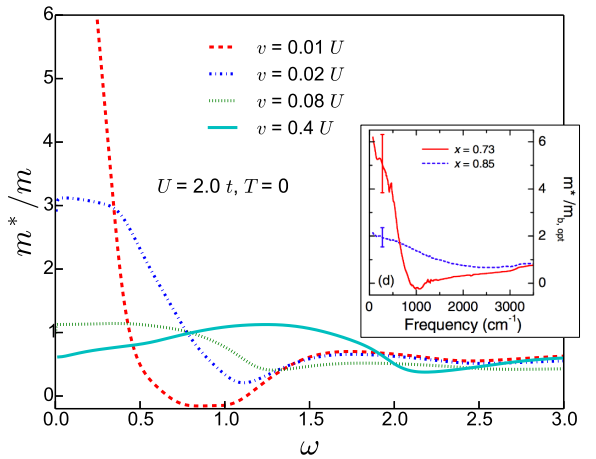

(a)

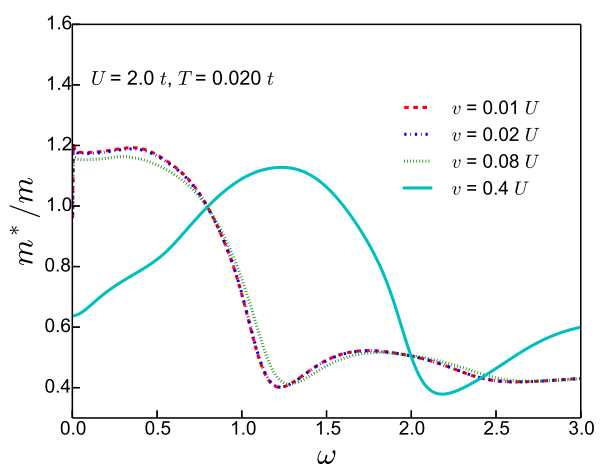

(b)

Fig. 4. Dynamical effective masses as functions of frequency $(U=2 t$. $)$ at (a) zero temperature and (b) finite temperature. At $v=0.4 U$, the Fermi-liquid property breaks down. The right inset in (a) shows similar experimental results reproduced from Ref. 7 .

related lattice and for fixed $t$, we mention four such important ones, viz. (i) Coulomb interaction $U$, (ii) carrier doping $\delta=1-n$, (iii) temperature $T$ and (iv) disorder potential $v$. At fixed $t$, decreasing $U$ lowers the ratio $t / U$ and effectively increases $\mathrm{BW}$. The width of the quasiparticle peak in the spectral density $A(\omega)$ represents a measurement of the effective BW. Fig. 5(a) shows the width enhances as $U$ is reduced from $2.6 t$ to $2.0 t$, however, keeping the height at the Fermi level $(\omega=0)$ unchanged due to the Luttinger pinning [28] property of a Fermi liquid. The pinning remains intact as well in the Drudé weight of optical conductivity $\left(\sigma_{1}(\omega \rightarrow 0)\right)$ though the mid-infrared peak position $\omega_{\text {peak }}$ acquires a blueshift and broadening as $U / t$ is reduced. Similarly increasing $T$ also broadens the quasiparticle peak though the Luttinger pinning does not hold any more. As a consequence of this, the Drudé peak in $\sigma_{1}(\omega)$ also diminishes. However, $\omega_{\text {peak }}$ redshifts as the $\mathrm{BW}$ increases due to temperature rise. Deviation from the particle-hole symmetry again leads to change in $\mathrm{BW}$. For small $\delta$, the Luttinger pinning is obeyed and effective BW increases as $\delta$ increases. Like in the $T$-driven case, BW increasing leads to redshift 


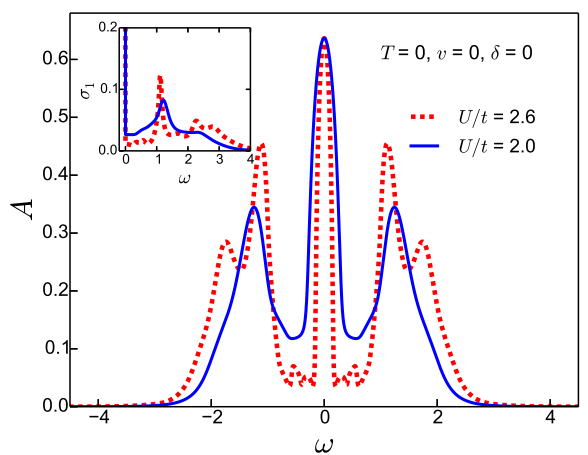

(a)

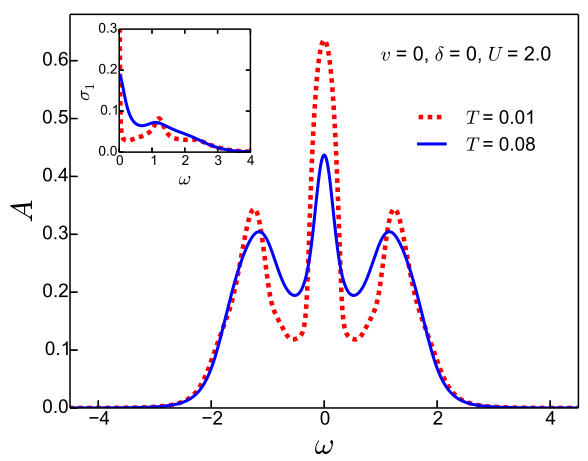

(b)

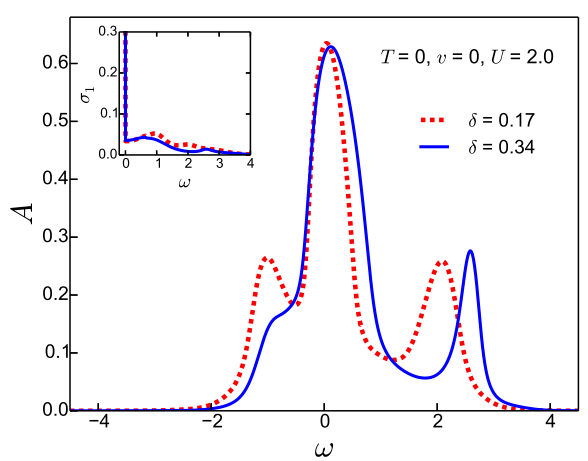

(c)

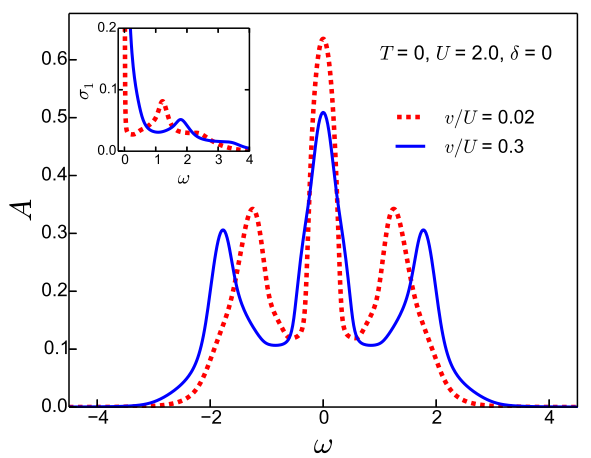

(d)

Fig. 5. Modification of spectral bandwidth due to (a) decrease in $U / t$, (b) increase in $T$, and (c) increase in doping $\delta=$ $1-n$, and increase in disorder potential $v$. The insets show corresponding changes in optical property $\sigma_{1}(\omega)$. in $\omega_{\text {peak }}$. Now if we look back to the calculations with changing disorder, we can see the absorption peak goes to a blueshift like the $U / t$-driven $\mathrm{BW}$ increase. This ensures that disorder indeed acts like chemical pressure from the change in BW perspective. The comparisons can be viewed in Fig. 5, which are summarized in the table below.

\begin{tabular}{|l|c|c|}
\hline Factor & Effect on BW & Effect on $\omega_{\text {peak }}$ \\
\hline \hline Pressure $(t / U)$ & Increase & Blueshift \\
\hline Disorder $(v / U)$ & Increase & Blueshift \\
\hline Temperature $(T)$ & Increase & Redshift \\
\hline Doping $(\delta)$ & Increase & Redshift \\
\hline
\end{tabular}

Summary : In this work, we for the first time establish the fact that the interaction/pressure and disorder driven bandwidth (BW) changes play very similar roles on optical properties, while other alternatives such as carrier filling and temperature change lead to different behaviors even though both shape the BW. Our results are at par with the the experiment on Br-doped BEDT conductor [7]. Though the experiment has been practically done in clean samples, our results invites similar experiments on disordered correlated systems [14]. Our investigation also could be generalized for a generic disorder distribution and to invoke the effect of Anderson localization the same DMFT formalism could be combined with the typical medium theory (TMT) [29]. On the DMFT side, thought IPT provides a reliable insight, recently developed exact impurity solvers such as continuous time Monte Carlo (CTQMC) method could be implemented to find more accurate results and compare to our predictions [30].

\section{ACKNOWLEDGMENTS}

HB owes to the DAE, Govt. of India for providing financial support and scientific resources. For the energy unit conversions we used the table provided by NIST, USA. 
* hbarhbar@gmail.com

$\dagger$ mslaad@imsc.res.in

¥ shassan@imsc.res.in

1 D. van der Marel et al., Nature 425, 271 (2003).

2 D. N. Basov and T. Timusk, Rev. Mod. Phys. 77, 721 (2005).

3 S. J. Moon et al., Phys. Rev. B 90, 014503 (2014).

${ }^{4}$ N. Barišić, D. Wu, M. Dressel, L. J. Li, G. H. Cao, and Z. A. Xu, Phys. Rev. B 82, 054518 (2010).

5 X. Deng, A. Sternbach, K. Haule, D. N. Basov, and G. Kotliar, Phys. Rev. Lett. 113, 246404 (2014).

6 M. M. Qazilbash, M. Brehm, B.-G. Chae, P.-C. Ho, G. O. Andreev, B.-J. Kim, S. J. Yun, A. V. Balatsky, M. B. Maple, F. Keilmann, H.-T. Kim, and D. N. Basov, Science 318, 1750 (2007).

7 J. Merino, M. Dumm, N. Drichko, M. Dressel, and R. H. McKenzie, Phys. Rev. Lett. 100, 086404 (2008).

8 S. Kamal, D. M. Kim, C. B. Eom, and J. S. Dodge, Phys. Rev. B 74, 165115 (2006).

9 H. T. Dang, J. Mravlje, A. Georges, and A. J. Millis, Phys. Rev. Lett. 115, 107003 (2015).

10 D. Geiger, U. S. Pracht, M. Dressel, J. Mravlje, M. Schneider, P. Gegenwart, and M. Scheffler, Phys. Rev. B 93 , 165131 (2016).

11 D. N. Basov, R. D. Averitt, D. van der Marel, M. Dressel, and K. Haule, Rev. Mod. Phys. 83, 471 (2011).

12 M. Imada, A. Fujimori, and Y. Tokura, Rev. Mod. Phys. 70, 1039 (1998).

13 M. c. v. M. Radonjić, D. Tanasković, V. Dobrosavljević, and K. Haule, Phys. Rev. B 81, 075118 (2010).

14 T. Sasaki, Crystals 2, 374 (2012).

15 M. S. Laad, L. Craco, and E. Müller-Hartmann, Phys. Rev. B 64, 195114 (2001).
16 K. Byczuk, W. Hofstetter, and D. Vollhardt, Phys. Rev. Lett. 94, 056404 (2005).

17 N. A. Kuleeva and E. Z. Kuchinskii, Journal of Experimental and Theoretical Physics 116, 1027 (2013).

18 A. I. Poteryaev, S. L. Skornyakov, A. S. Belozerov, and V. I. Anisimov, Phys. Rev. B 91, 195141 (2015).

19 C. E. Ekuma, S.-X. Yang, H. Terletska, K.-M. Tam, N. S. Vidhyadhiraja, J. Moreno, and M. Jarrell, Phys. Rev. B 92, 201114 (2015).

20 A. Georges, G. Kotliar, W. Krauth, and M. J. Rozenberg, Rev. Mod. Phys. 68, 13 (1996).

${ }^{21}$ H. Barman and N. S. Vidhyadhiraja, Int. J. Mod. Phys. B 25, 2461 (2011).

22 B. Velický, S. Kirkpatrick, and H. Ehrenreich, Phys. Rev. 175, 747 (1968).

${ }^{23}$ H. Barman, , M. S. Laad, and S. R. Hassan, "Realization of a Two Relaxation Rates in the Hubbard-FalicovKimball Model," (2016), arXiv:cond-mat/1611.07594 [cond-mat.str-el].

24 T. Pruschke, M. Jarrell, and J. Freericks, Ann. Phys., Lpz. 44, 187 (1995), http://dx.doi.org/10.1080/00018739500101526.

25 E. Lange and G. Kotliar, Phys. Rev. B 59, 1800 (1999).

26 C. Berthod, J. Mravlje, X. Deng, R. Žitko, D. van der Marel, and A. Georges, Phys. Rev. B 87, 115109 (2013).

27 D. Stricker, J. Mravlje, C. Berthod, R. Fittipaldi, A. Vecchione, A. Georges, and D. van der Marel, Phys. Rev. Lett. 113, 087404 (2014).

28 D. Vollhardt, K. Held, G. Keller, R. Bulla, T. Pruschke, I. A. Nekrasov, and V. I. Anisimov, J. Phys. Soc. Japan 74, 136 (2005).

${ }^{29}$ V. Dobrosavljevi, A. A. Pastor, and B. K. Nikoli, EPL (Europhysics Letters) 62, 76 (2003).

30 E. Gull, A. J. Millis, A. I. Lichtenstein, A. N. Rubtsov, M. Troyer, and P. Werner, Rev. Mod. Phys. 83, 349 (2011) 